might be paying the full price for parallelly imported material and have to pay more for other products, because of the profit control scheme.

So what of the future? The present state of affairs is not in the nation's interest, nor will it benefit the public. Apparently Mr Norman Fowler, Secretary of State for Social Services, intends to introduce a new Medicines Order within the next few weeks at the same time as the modified licensing system comes into operation. ${ }^{3}$ The new system is intended to ensure that importations will be restricted to small quantities of unlicensed medicinal products or that the products are entirely satisfactory in every aspect. The proposals provide that, firstly, parallel imports must be licensed in a European Economic Community member state and have no different therapeutic effect from the product already licensed in Britain. The Licensing Authority will be able to check that the product is effectively covered by a British product licence. Conditions of storage, distribution, packaging, labelling, and inspection are all covered by the order.

The second stipulation concerns the people who at present are acting as wholesalers and on occasions supplying prescription only medicines to non-pharmacists. In future such people will require a wholesale dealer's licence. In instances where the products need relabelling the parallel importer will require a manufacturer's (assembly only) licence. The new order will make it illegal to advertise, and this should stop the practice of unsolicited material being sent through the post. Arrangements for batch control information and product recalls are also included.

Since several other countries have already taken action to protect themselves these proposed new measures are long overdue (Association of the British Pharmaceutical Industry, consultative document MLX 150). Only time will tell if they are effective.

Regional Pharmaceutical Officer,

North West Thames Regional

Health Authority,

London W2 6AF

${ }^{1}$ Clarke K. Parliamentary written answer. House of Commons Official Report (Hansard) 1984 January $24 ; 52:$ col 556 . (No 84.)

2 Pharmaceutical Society Council. Supply of medicines-use of nonEnglish labels. Pharmaceutical fournal 1984;232:292.

${ }^{3}$ Fowler N. Parliamentary written answer. House of Commons Official Report (Hansard) 1984 April 5;57:cols 647-8. (No 135.)

\section{Dilate the pupil and see the fundus}

No ophthalmologist expresses an opinion on a fundus through an undilated pupil-a generalisation with few exceptions. The theoretical risk that mydriatic drugs may produce acute closed angle ("congestive") glaucoma has been overestimated.

Tropicamide $0.5 \%$ eye drops from sterile single dose capsules are almost ideal, because they produce a useful effect in about 20 minutes, which rises to a peak at 40-60 minutes; the effect passes off in six to eight hours. ${ }^{1}$ Cyclopentolate $1 \%$ and homatropine $1 \%$ are less useful, because their action lasts about 24 hours. ${ }^{1}$ The effect of atropine lasts for a week or more so it is rarely used for viewing the fundus. ${ }^{1}$ The individual response to all these drugs varies and is poorest in the dark brown iris. They act by weakening the sphincter muscle of the pupil: they are parasympatholytic. The tone of the dilator muscle, under sympathetic control, then enlarges the pupil; that action may be supplemented by the powerful adrenergic phenylephrine $10 \%$ to achieve wide mydriasis. ${ }^{2}$ Phenylephrine alone works moderately well with the same time relations as tropicamide. ${ }^{1}$

The mydriasis may be reversed within about an hour with pilocarpine $0.5 \%$ or $1 \%$, which directly stimulates the muscle fibres of the pupillary sphincter. Many ophthalmologists omit that, however, because of the discomfort of strong miosis and spasm of the ciliary muscle (accommodation), preferring to allow the effect of mydriatics to decline with time. Thymoxamine $0.5 \%$ will selectively eliminate sympathetic stimulation only, and so is a precise way to reverse the effect of phenylephrine. ${ }^{3}$

Without mydriasis the clinician will see only the optic disc reasonably well - at least in the elderly, because the older the patient the smaller the pupil. The very important macula and fovea are particularly difficult to see because stimulation of that area by the light of the ophthalmoscope will cause maximal constriction of the pupil through the pupillary light reflex arc. Evaluation of early cataract usually requires mydriasis. Some of the mysteries of medical retinopathies can be dispelled by the physician's seeing the fundus through a dilated pupil, especially if he has raised his probability of correct diagnosis by testing the patient's urine for sugar and recording the blood pressure beforehand.

The risk of producing acute closed angle (congestive) glaucoma is small. In a predisposed eye, dilatation of the pupil causes the peripheral iris to block the trabecular meshwork, the escape route for aqueous humour. This leads to a sudden rise in intraocular pressure and speedy loss of vision from corneal oedema ("steamy cornea"). The doctor should be wary of the patient with small eyeballs, corneas of small diameter, and shallow anterior chambers, especially if a parent or sibling is known to have glaucoma or has been admitted to an eye hospital for an emergency operation for glaucoma. A simple test may help to show that the pupil is on a more anterior plane than the periphery of the iris: shine a bright light with a concentrated beam from the temporal side along the plane of the iris. ${ }^{4}$ If the nasal side of the iris is in shadow that suggests a shallow anterior chamber. If in doubt, dilate only one pupil with phenylephrine $10 \%$ and reverse its effect by thymoxamine $0.5 \%$, leaving examination of the other eye for a subsequent visit. ${ }^{56}$

Other ocular side effects should be remembered. The patient will have some difficulty in reading with the treated eye(s) for the rest of the day unless his presbyopia is complete and he has full correction with spectacles. The explanation is that the ciliary muscle (for accommodation) is affected as well as the pupil. A chauffeur must be available to drive the patient home, since a car accident could be caused by glare when the sun is low in the sky.

Systemic toxic effects are possible from drugs in eye drops which sidestep initial detoxification by the liver, ${ }^{7}$ though these are rare when a small single dose is used. ${ }^{89}$ The patient may notice the taste of the drug as it drains into the nose and pharynx through the nasolacrimal duct, adding to the absorption by conjunctival blood vessels. Children and the elderly are most at risk. As might be expected, adrenergics may cause cardiac irregularities, with raised blood pressure, headache, trembling, and sweating; all these features may be worse in patients taking tricyclic antidepressants or monoamine oxidase inhibitors or being given halothane. ${ }^{10}$ The parasympatholytics tend to produce effects on the central nervous system, from bad dreams to confusional psychosis. ${ }^{711}$ 
The cholinergic drug pilocarpine may cause sweating and salivation, nausea, vomiting and diarrhoea, and bronchospasm.

Finally, any mydriatic at all used by a casualty officer in an unconscious patient will produce a severe systemic adrenergic reaction-in the neurologist or neurosurgeon who receives the patient. The casualty officer should never forget that the size and reactions of the pupils are important in the management of patients in coma, with head injuries, or with many other neurological diseases.

Professor of Ophthalmology,

C I PHILlips

University of Edinburgh,

Princess Alexandra Eye Pavilion,

Edinburgh EH3 9HA

1 Smith SE. Mydriatic drugs for routine fundal inspection. A reapraisal. Lancet 1971;ii:837-9. Apt L, Henrick A. Pupillary dilatation with single eye drop mydriatic combinations. Am $\mathcal{J}$ Ophthalmol 1980;89:553-9.

${ }^{3}$ Mapstone R. Safe mydriasis. Br f Ophthalmol 1970;54:690-2.

Becker B, Shaffer RN. Diagnosis and therapy of the glaucomas. 2nd ed. St Louis: C V Mosby Co, 1965:149-50.

${ }_{6}^{5}$ Mapstone R. Dilating dangerous pupils. Br $\mathcal{f}$ Ophthalmol 1977;61:517-24

Saheb NE, Lorenzetti D, East D, Salpeter-Carlton S. Thymoxamine versus pilocarpine in the reversal of phenylephrine-induced mydriasis. Can f Ophthalmol 1982;17:266-7.

${ }^{7}$ Davidson SI. Systemic effects of eye drops. Trans Ophthalmol Soc UK 1974;94(part II):487-95.

${ }^{8}$ Meyer SM, Fraunfelder FT. Phenylephrine hydrochloride. Ophthalmology 1980;87:1177-80.

Brown MM, Brown GC, Spaeth GL. Lack of side-effects

${ }^{10}$ Davidson SI. Drug interactions in ophthalmology. Trans Ophthalmol Soc UK 1975 .95 (part II) 277 80.

${ }^{11}$ Asher R. A taste of Asher. London: Keynes Press, 1983:23.

\section{Autoimmune thyroid disease and pregnancy}

The care of a woman with autoimmune thyroid disease who becomes pregnant presents two problems: the effect which the maternal disease may have on the fetus and newborn, and the influence which pregnancy itself may have on the maternal disease.

A successful normal pregnancy assumes the adjustment of the mother's immune system to an antigenically foreign fetoplacental unit. For reasons still uncertain the maternal immune response is generally suppressed progressively through pregnancy. 1 This is manifested by a decreased blastogenic response of mitogen stimulated lymphocytes, a fall in the serum concentration of the IgG immunoglobulins, and alterations in the numbers of peripheral blood $\mathrm{T}$ lymphocyte series variably reported as an increase in suppressor $\mathrm{T}$ cells or a decrease in helper $\mathrm{T}$ cells. Though such changes seem unlikely to reflect primary events, the factors responsible remain to be determined. One possibility is that fetal suppressor $\mathrm{T}$ cells or their soluble products may help modulate the maternal immune response.

Against that background, how does pregnancy affect autoimmune thyroid disease? In normal pregnancy maternal IgG crosses the placenta and is detectable in the fetal and neonatal circulation, where it provides a protective mechanism until the neonate can generate his own IgG response to foreign challenge. Since maternal IgG crosses the placenta any autoantibodies of this immunoglobulin subclass will do likewise. Several thyroid autoantibodies can influence fetal and neonatal thyroid function. In hyperthyroid Graves' disease antibodies to the thyrotrophin receptor stimulate thyroid function. ${ }^{2}$ Such antibodies may persist even after thyroidectomy or treatment with radioiodine. If present in high titre in the third trimester of pregnancy ${ }^{3}$ they may stimulate the fetal thyroid and cause intrauterine hyperthyroidism. ${ }^{4}$ If the mother is receiving an antithyroid drug such as carbimazole this may also cross into the fetus and prevent hyperthyroidism-but the active immunoglobulin may persist for a month or two in the child's circulation and then cause a self limiting neonatal hyperthyroidism. This condition requires treatment with antithyroid drugs. ${ }^{6}$

More recently blocking antibodies interacting with the thyrotrophin receptor have been recognised in autoimmune thyroid disease. These antibodies block the action of thyrotrophin on the thyroid and so can be responsible for hypothyroidism in some patients with myxoedema and Hashimoto's disease. During pregnancy such antibodies cross the placenta and are one cause, albeit rare, of intrauterine and neonatal hypothyroidism; this remits spontaneously as the maternal immunoglobulins disappear from the child's circulation.? Combinations of stimulating and blocking antibodies may interact with the fetal and neonatal thyroid, and-depending on their affinities and relative titres-lead to varying alterations of thyroid function-for example, hypothyroidism progressing to hyperthyroidism, which then remits. ${ }^{89}$

The clinical message is that serum concentrations of thyrotrophin receptor antibodies should be checked in the third trimester of pregnancy in all women with autoimmune thyroid disease-namely, Graves' disease, Hashimoto's disease, and myxoedema. Several assays are available, but the most convenient and widely available is that depending on the inhibition of labelled thyrotrophin binding to solubilised thy otrophin receptors by the receptor antibodies. ${ }^{10}$ Children born to mothers with high titres of thyrotrophin receptor antibody are at risk of developing one of the neonatal thyroid syndromes.

Pregnancy also has profound effects on the autoimmune thyroid diseases. Studies from both Japan and Norih America have shown changes in clinical thyroid state and in biochemical and serological measurements during pregnancy and after birth. In a study of 41 pregnancies in 35 patients with Graves' disease in remission Amino et al showed recurrence of disease in early pregnancy and after delivery and amelioration in the latter half of pregnancy. ${ }^{11}$ They had previously shown a fall in circulating concentrations of thyroglobulin and microsomal antibodies during pregnancy followed by rises post partum in patients with Graves' disease and autoimmune thyroid disease. ${ }^{12}$ Similar changes in antibody concentrations have been seen in pregnancies in normal women. ${ }^{13}$ In the United States Turney et al found postpartum thyroid disease, usually autoimmune, in $9 \%$ of 238 women. ${ }^{14}$ Likewise, Amino and his colleagues found that $5.5 \%$ of 507 postpartum Japanese women had transient hyperthyroidism or hypothyroidism three to eight months after delivery. ${ }^{15}$ No similar studies have been reported from Britain. Postpartum autoimmune thyroid disease has distinctive characteristics: a high frequency of previous goitre; early (at less than four months) goitre or hyperthyroidism or both, and later (over four months) hypothyroidism, sometimes in the same patients; a return to euthyroidism five to 10 months post partum associated with decrease in size but occasional persistence of the goitre; and increasing titres of thyroid microsomal antibodies, peaking at three to four months. ${ }^{16}$

The basis of postpartum hyperthyroidism remains uncertain. A few patients appear to have Graves' disease with an increased radioiodine uptake by the thyroid, but most show the suppressed uptake of the postpartum painless thyroiditis with transient thyrotoxicosis-the PPTT syndrome described by Ginsberg and Walfish. ${ }^{17}$ Whether this syndrome is autoimmune or viral is still unknown, though it is very similar to 